\title{
Current Capacity Limits and Activities within the EU Project MODE-GAP to Overcome Them
}

\author{
A.D.Ellis \\ Tyndall National Institute and Department of Physics, University College Cork, Cork, Ireland \\ Phone: +353214904858 Email: andrew.ellis@tyndall.ie
}

\begin{abstract}
In this presentation we will discuss the implications of the so called Nonlinear Shannon Limit. We will compare technologies including new fibres for long haul transmission and techniques to expand the capacity of existing standard single mode fibres.

\section{Capacity Limits}

Current research results, based on DSP enabled coherent detection, are rapidly approaching the fundamental capacity limits of conventional optical fibres ${ }^{1}$, see Figure 1. $100 \mathrm{Gbit} / \mathrm{s}$ coherent products are now available from a range of competing system and module vendors. In addition to optimizing the utilization of available fiber capacity $^{2}$, research is also shifting towards adaptive and intelligent optical systems ${ }^{3}$. These require complex trade-offs between single and multi-carrier techniques, and offer flexibility in modulation formats ${ }^{4}$ and symbol rates $^{5}$. From a networking point of view, the focus is on the maximization of the overall information throughput based on the layout of the network itself ${ }^{6}$, and the adoption of flexi-grid alternatives to ITU based reconfigurable add drop multiplexers? ${ }^{7}$.
\end{abstract}

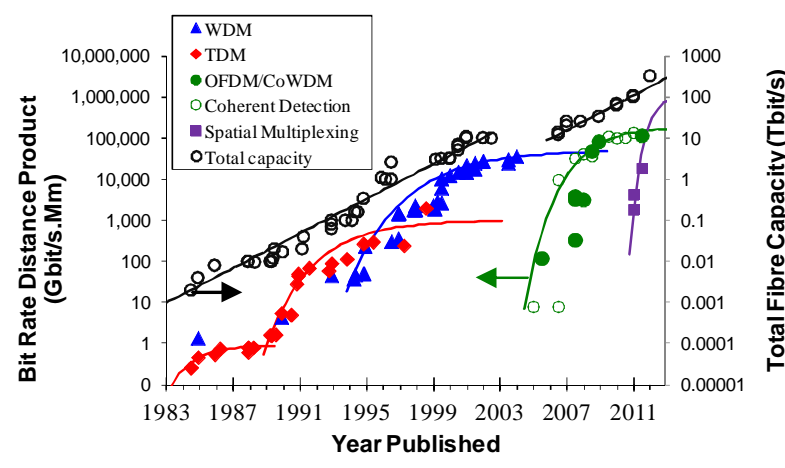

Figure 1; Evolution of record transmission experiments showing bit rate distance product (left axis, coloured symbols denoting technologies) and total bit rate (black symbols)

Figure 1 illustrates two key points. Firstly, there was a distinct pause in progress from 2002 to 2006 corresponding to the end of the "dot com bubble". Secondly, it illustrates that whilst headline total "fibre" capacities have resumed their former exponential growth trend, overall system performance including reach (expressed as bit rate distance product) does not appear to have resumed its former growth rate, with coherent detection adding less than an order of magnitude to the overall capacity ${ }^{9}$ and a clear gap is growing between the two curves. It is widely believed that the decline in the growth is due to the so called nonlinear Shannon limit, where an optimum launch power balancing the impact of noise and inter channel nonlinearities exists ${ }^{10}$. Although recent reports suggest that under certain circumstances, the nonlinear channel capacity should continue to increase with increasing signal launch power ${ }^{11,12}$ numerical and experimental results with specific modulation formats currently support the nonlinear Shannon limit formulation for WDM systems ${ }^{13}$.

For a 2,000 km system, the nonlinear Shannon limit for a conventional single mode fiber is in the region of 5 $\mathrm{bit} / \mathrm{s} / \mathrm{Hz} / \mathrm{pol}$, giving a maximum fibre capacity of the order of $50 \mathrm{Tbit} / \mathrm{s}$ in the $\mathrm{C}$ band. It is often assumed that the bandwidth demand will continue its uninterrupted $40 \%$ compound annual growth experienced since optical systems were first deployed in 1975. Consequently if several $100 \mathrm{Gbit} / \mathrm{s}$ line cards are installed simultaneously today to upgrade link capacity in response to demand, similar new deployments in 10 years time would be of fully lit fibres. Widespread cable deployments will be required shortly after this. Since the various techniques to optimise the utilisation of the fiber capacity, including compensation of inter channel nonlinearity, only offer incremental benefit, major research programs such as EXAT and MODE GAP are examining novel optical fibres. The fibres may be divided into two broad categories, those enabling spatial multiplexing, such as multi-mode fibers and multi core fibres, and those aimed at radically reducing the loss and nonlinear coefficients of the fibres.

Figure 2 illustrates the nonlinear Shannon limit for a variety of optical fibre proposals for two repeater spacings. Whilst the use of a new fibre always improves the information spectral density when compared to the conventional single mode fibre (solid black line), the choice between ultra low loss hollow core fibres and spatial multiplexing is critically dependent on the system configuration. Furthermore, raw information carry capability is not the whole story, and the energy consumption and cost of the proposed solution are different. For example, to achieve an ISD of $10 \mathrm{~b} / \mathrm{s} / \mathrm{Hz}$ (PM-32QAM), the hollow core fibre requires a per amplifier launch power of $14.5 \mathrm{~mW}$ at $200 \mathrm{~km}$, but only $7 \mathrm{~mW}$ with a $100 \mathrm{~km}$ spacing. However the total number 
of amplifiers is changed and the sum of the required output power over all amplifiers is almost constant. This is not the case for the multimode fibre, where a $16 \mathrm{~dB}$ higher total launch power is required to increase the amplifier spacing from 100 to $200 \mathrm{~km}$.
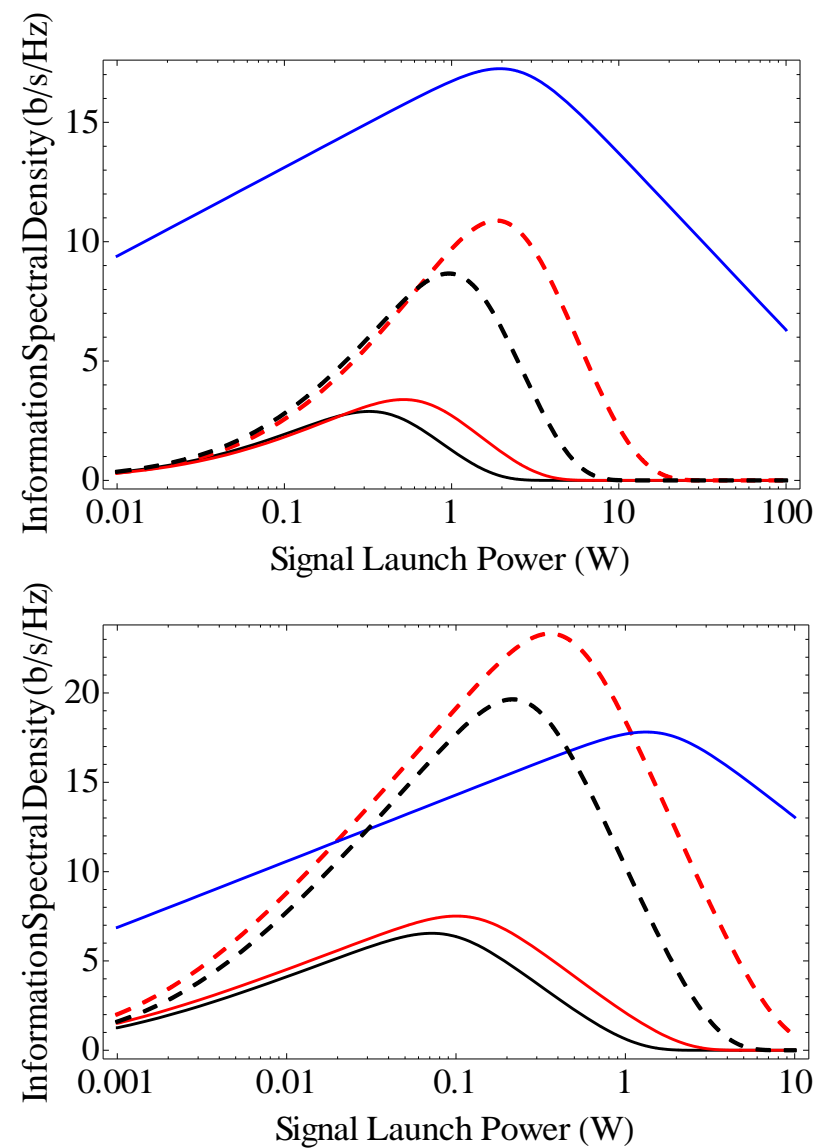

Figure 2: Nonlinear Shannon limit for a $2 \mathrm{THz}$ bandwidth $2,000 \mathrm{~km}$ transmission system with $200 \mathrm{~km}$ repeater spacing (top) and $100 \mathrm{~km}$ repeater spacing (bottom) for ultra low loss hollow core fibre operated single mode (blue), multi core fibre (black) and multi-mode fibre (red). Spatially multiplexed fibres shown with 1 (solid) or 3 (dashed) modes. Fibre losses are $0.05,0.2$ and $0.22 \mathrm{~dB} / \mathrm{km}$ respecively, nonlinear coefficents $0.0014,1.4$ and $\sim 0.4$ (varies with mode) $/ \mathrm{W} / \mathrm{km}$ respectively.

During the presentation, we will examine the consequences of these energy tradeoffs in more detail, including estimates of the relative impact of fibre and transponder designs.

\section{MODE GAP}

Within MODE-GAP both approaches are studied, spatial multiplexing using solid core multi-mode fiber ${ }^{14,15}$ and ultra low loss hollow core fibre ${ }^{16}$. In addition to activities in direct fibre design, overall system design, capacity limits $^{17}$ and nonlinear propagation ${ }^{18}$, MODE GAP is also developing the necessary ancillary technologies. This includes fibre based ${ }^{19},{ }^{20}$ and planar mode multiplexers, digital signal processing algorithms ${ }^{21}$, and modal gain optimised fibre amplifiers ${ }^{22}$. Mode multiplexers offer extinction ratios in excess of $20 \mathrm{~dB}$ over a $40 \mathrm{~nm}$ wavelength range. Our studies of the complexities of digital signal processing show a clear advantage to using the cyclic prefix in an OFDM formatted signal to overcome the impulse response of a multi-mode fibre, but also clearly demonstrate that the maximum practical differential mode delay is limited, leading to the development of mode delay compensation techniques ${ }^{23}$.

Similarly, MODE GAP is developing the necessary ancillary technologies for operation with hollow core fibre including lasers optimised for use at the predicted loss minima of the hollow core fibre ${ }^{24}$, couplers, modulators, amplifiers and photodiodes ${ }^{25}$.

\section{Conclusions}

This presentation will analyse the prospects for increasing the capacity of optical communication networks assuming the deployment of new optical fibre cables. It will discuss the optimum fibre parameters from the view point of both total capacity and energy consumption and will summarise the progress towards implementing communication systems based on new fibre types in the EU project MODE-GAP.

\section{Acknowledgements}

This material is based upon work supported by Science Foundation Ireland under Grant 06/IN/I969 and by the European Communities 7th Framework Programme grant 258033 (MODE-GAP).

\section{References}

1 A.D.Ellis et al., JLT, 28, 4, pp 423 (2010)

2 X.Liu et al, OFC 2012, paper PDP5B.3 (2012)

3 L.Liu et al., OFC 2012, paper PDP5D.2 (2012)

4 W. Freude et al., ICTON 2011, paper TuD1.1 (2012)

5 A.D.Ellis, et al., IEEE Sum.Top., paperTuD3.2, (2009)

6 C.K.Kallo et al., NOC 2011, paper TPN-7 (2011)

7 J. Rahn et al., OFC 2012, paper PDP5D5 (2012)

8 L.Gomes, Wall Street Journal, 8/11/2006, ppB1 (2006)

9 M-F. Huang et al., OFC 2011 paper PDP.B5 (2011)

10 P.P.Mitra et al.,, Nature, 411, pp 1027-1030, (2001)

11 S.K.Turitsyn et al, forthcoming, ICTON 2012 (2012)

12 E. Agrell, forthcoming, arXiv:1108.0391v2 (2012)

13 P.Poggiolini et al., PTL, 23, 11, pp742, (2011)

14 K. Jespersen et al., OFC 2012, paper OTh3I.4, (2012)

15 L. Gruner-Nielsen et al, OFC, paper PDP5A1 (2012)

16 N.V.Wheeler et al. OFC paper PDP5A2 (2012)

17 A.D.Ellis et al,, IEEE IPC, paper TuN1, (2011).

18 N.MacSuibhne et al., submitted to OECC 2012.

19 I.P.Giles et al., IEEE Sum.Top, invited paper, (2012)

20 L. Grüner-Nielsen et al., Submitted to IEEE Sum.Top (2012)

21 A.P.L.Polo et al., OFC, paper OTu2C2, (2012)

22 Y. Yung et al., ECOC, paper Th.13.K.4 (2011)

23 B. Inan et al., forthcoming ICTON 2012 (2012)

$24 \mathrm{http}: / / \mathrm{www}$.eblanaphotonics.com/products-specialitydiode-lasers.php

25 http://www.phoenixphotonics.com/website/products/ 2microncouplers.htm 